\title{
Feasible Delivery System for Eradicating Ebola
}

\section{Cui Wang}

School of North China Electric Power University, Baoding 071000, China;

1543025473@qq.com

Keywords: multi-objective optimization, non-linear constrains, delivery network

\begin{abstract}
Ebola virus disease is a severe, often-fatal illness and has a case fatality rate up to $90 \%$. Ebola crisis could severely do harm to the economies of Guinea, Liberia and Sierra Leone unless action is taken quickly. In order to eradicate the disease with the shortest time and the least cost, we build a feasible delivery system. We abstract our delivery system into a network, where points is place to store medication and edges is transport lines. This is a multi-objective optimization problem which is related to the demand of the medication and the least cost. Based on the reality,we establish a target equation to minimize expenditure, taking the amount of the medication needed and offered as non-linear constrains. Besides, we define an algorithm to measure the amount of medication distributed in different points and get the amount of the medication to deliver in different two points.
\end{abstract}

\section{Introduction}

In order to establish a reasonable drug delivery system, we need to consider the amount of drug, the shortest distance and minimization of transportation costs. This is a multi-objective programming problem ${ }^{[1]}$. We divided our transportation system into supply points, demand points and transport lines. Taking into account the traffic situation, we choose the airplane as our means for transport.

\section{Assumptions}

The transport system is affected by various factors ,which is far too complex. We make the following assumptions to approximate and simplify the problem.

- Assume that transport distance between the supply points and storage points is a straight line distance.

- The specific location of supply points and demand points is related to the distribution of airport and patients, we assume that the position has been determined.

\section{Model Building}

We defined an algorithm to measure the amount of medicine distributed in each point $\mathrm{M}_{\mathrm{ij}}$. Which can be defined as follows:

$$
M_{i j}=\sum_{i}^{m} \frac{d_{j}}{s_{i j}}
$$

Where $m$ is the total number of points to store medicine, $d_{j}$ is the number of medicine in $\mathrm{j}$, $s_{i j}$ is the number of medicine transport between $\mathrm{i}$ and $\mathrm{j}$.

Based on the limited data ${ }^{[2]}$, we can get the contact between the number of patients and time. $B(t)=\left\{\begin{array}{l}28111-1.1287 t^{2}+140.3 t(0 \leq t \leq 28) \\ 28111+(-1.1287-0.5 x) t^{2}+(140.3+28 x) t-392 x\left(28 \geq t>t_{0}\right)\end{array}\right.$ 
The equation target function:

$$
f=\sum_{i}^{m} \sum_{j}^{n} x_{i j} s_{i j} p
$$

Where $m$ is the total number of points to store medicine, $n$ is the total number of the points which need medicine, $\mathrm{f}$ is the total cost, $\mathrm{p}$ is the cost on delivering medication for a patient need in unit of distance.

Constraint conditions:

$$
\left\{\begin{array}{l}
\sum_{j=1}^{m} x_{i j} \leq c_{i} \\
\sum_{i=1}^{n} x_{i j} \geq d_{j}
\end{array}\right.
$$

Where $d_{j}$ is the number of medicine in $\mathrm{j}, c_{i}$ is the number of medicine in $\mathrm{i}$.

\section{Model solving}

Because the medication to treat the Ebola disease is high of technology and science, there is no ability to produce it in these economically backward countries. So, we must build some storehouses to stockpile medications. From the available data ${ }^{[3]}$,we calculate the number of medicine stored in three countries' international airport, where patients are also clustered.

Table2 the number of medicine stored in three airport

\begin{tabular}{|l|l|l|l|}
\hline Airport & 1 & 2 & 3 \\
\hline Faranah(1) & 80 & 319 & 678 \\
\hline Freetown lungi(2) & 519 & 239 & 798 \\
\hline Monrovia Roberts intl(3) & 758 & 519 & 180 \\
\hline
\end{tabular}

We can calculate the amount of medicine distributed in each airport.

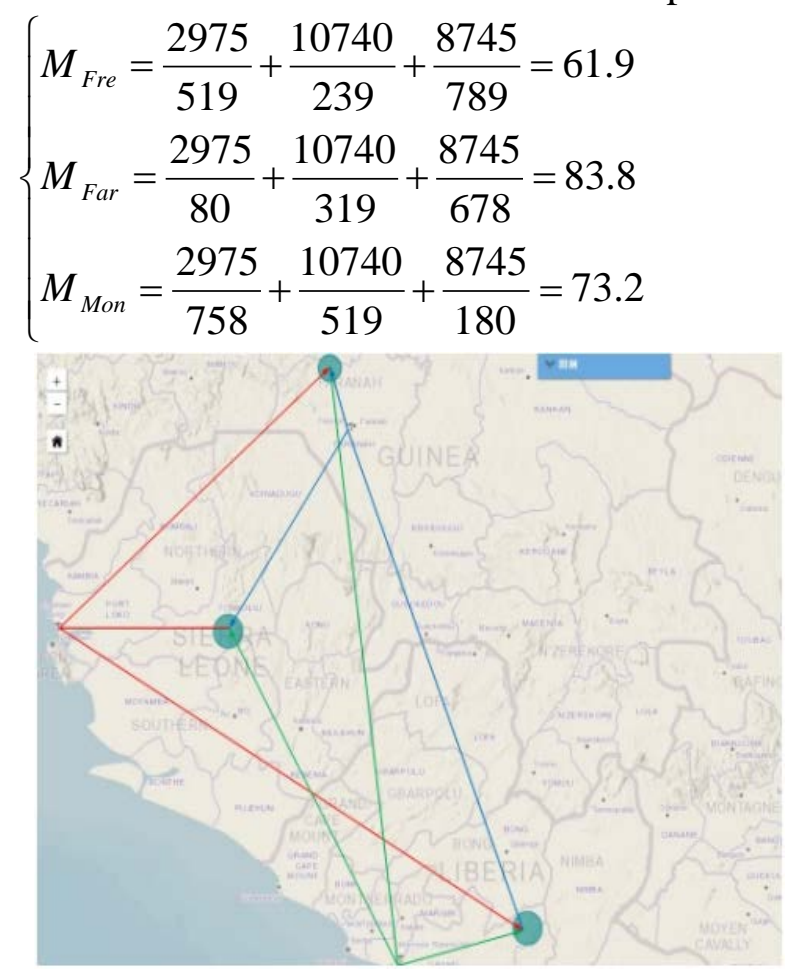

Figure 1.The storing points and transport lines in three countries 
The storing points and transport lines in three countries can be clearly showed in Figure 1 . We get target function is:

$f=160 x_{11}+638 x_{12}+1356 x_{13}+1038 x_{21}+478 x_{22}+1596 x_{23}+1516 x_{31}+1038 x_{32}+360 x_{33}$

Constraint conditions are:

$$
\left\{\begin{array}{l}
x_{11}+x_{12}+x_{13} \leq 8759 \\
x_{21}+x_{22}+x_{23} \leq 6289 \\
x_{31}+x_{32}+x_{33} \leq 7412 \\
x_{11}+x_{21}+x_{31} \geq 2975 \\
x_{12}+x_{22}+x_{32} \geq 10740 \\
x_{13}+x_{23}+x_{33} \geq 8745
\end{array}\right.
$$

With lingo we can get the optimal solution:

$$
\left\{\begin{array}{l}
x_{11}=2975 \\
x_{12}=4451 \\
x_{13}=1333 \\
x_{22}=6289 \\
x_{33}=7412 \\
x_{21}=x_{23}=x_{31}=x_{32}=0 \\
f=1.07977 \times 10^{7}
\end{array}\right.
$$

Where $x_{\mathrm{ij}}$ is the amount of the medicine delivered between two points.

\section{Conclusion}

We built a delivery system and get air-traffic volumes for medicine transporting in different disaster areas. We applied it in Guinea, Liberia and Sierra Leone for eradicating Ebola disaster and get exactly amount of the medicine delivered in different airport with the least cost.

\section{References}

[1] Miaocheng, Xuweisheng, Wuqidi, Construction and solving large-scale emergency relief supplies transport model. Systems Engineering(November 2006)

[2] http://zh.wikipedia.org/wiki

[3] http://maps.who.int/MapJournal 